\title{
Strategisches Personalmanagement in der öffentlichen Verwaltung im Zeichen des demografischen Wandels
}

\author{
Florian Kunze
}

Der demografische Wandel ist eine der großen Herausforderungen, insbesondere für Organisationen der öffentlichen Hand. Schon heute sind sie mit einem stark steigenden Durchschnittsalter ihrer Belegschaften konfrontiert, das eine zuverlässige Dienstleistungserstellung in Zukunft in Frage stellt, da sowohl die körperliche Konstitution, die Motivation als auch die Qualifikation in einer alternden Belegschaft rückläufig sein können. Zur Bewältigung dieser Herausforderung ist eine Professionalisierung des Personalmanagements in den öffentlichen Verwaltungen unerlässlich. Dieser Beitrag stellt deshalb dar, wie ein strategisches Personalmanagement in den Bereichen präventives Gesundheitsmanagement, Personalentwicklung, lebenslanges Lernen und einen lebensphasenorientiertes Karrieremanagement zum erfolgreichen Umgang mit dem demografischen Wandel in der öffentlichen Verwaltung gestaltet werden kann.

\section{Der demografische Wandel als Herausforderung für die öffentli- che Verwaltung}

Der demografische Wandel ist eine der zentralen gesellschaftlichen und politischen Herausforderung unserer heutigen Zeit. Durch die steigende Lebenserwartung und fallende Geburtenquoten findet derzeit eine massive Verschiebung im Durchschnittsalter und in der Bevölkerungszusammensetzung in vielen westlichen Industrieländern und insbesondere in Deutschland statt. Diese Entwicklung hat auch starke Auswirkungen auf die Struktur und die Alterung der Erwerbsbevölkerung. Der öffentliche Dienst, mit 4,6 Millionen Beschäftigten der größte

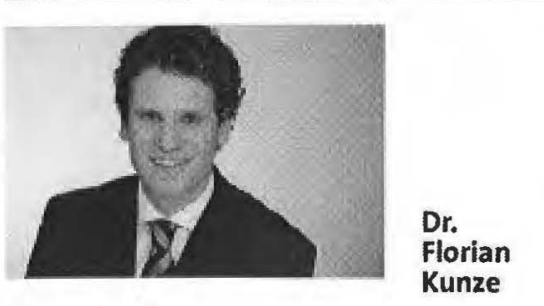

Wissenschaftlicher Projektleiter und Lehrbeauftragter
Arbeitgeber in Deutschland', ist hiervon in besonderen Maße betroffen. So ist von 2000 bis 2010 das Durchschnittsalter der Beschäftigten in allen öffentlichen Verwaltungen von 42,3 auf 44,6 Jahre gestiegen und auch der Prozentsatz der über 55-jährigen Beschäftigten hat um mehr als 8 Prozentpunkte auf 23 Prozent zugenommen. ${ }^{2}$ Wie Abbildung 1, die die Altersstruktur der Beschäftigten des öffentlichen Dienstes mit der Altersstruktur der sonstigen sozialversicherungspflichtigen Beschäftigen vergleicht, deutlich macht, dominieren in der öffentlichen Verwaltung schon heute die beiden ältesten Altersgruppen (45-55 Jahre, 55 Jahre und älter). Insofem sind zukünftige Szenarien einer alterszentrierten Belegschaft, die für den privaten Sektor erst in ca. 20 Jahren prognostiziert werden, für die öffentliche Verwaltung schon heute Realität (vgl. Abb. 1 auf der nächsten Seite).

Für die öffentliche Verwaltung bedeutet dies, dass sie akute Anstrengungen unternehmen muss, un die Leistungsfähigkeit ihrer alternden Belegschaft zumindest zu erhalten und nach Möglichkeit noch auszubauen, um in Zeiten schwindender finanzieller Spielräume der öffentlichen Hand weiterhin handlungsfähig in der Dienstleistungserstellung $\mathrm{zu}$ sein. Umso erstaunlicher ist es, dass in öffentlichen Bereich immer noch das Paradigma einer passiven Personalverwaltung gegenüber einem aktiven Management der Ressource Personal dominiert. ${ }^{3}$ Zwar wird die Alterung des Personals von Führungskräften, zum Beispiel im kommunalen Bereich, als ein zentrales Handlungsfeld angesehen, aber Umsetzungen in Form eines proaktiven strategischen Personalmanagements sind in der Praxis kaum zu erkennen. ${ }^{5}$ In dem vorliegenden Beitrag soll es deshalb darum gehen, zentrale Bestandteile eines strategischen Personalmanagements für die offentliche Hand in Zeichen des demografischen Wandels zu erläutern. Hierfür werden im folgenden Abschnitt zunächst die drei Herausforderungen für ein solches Personalmanagement aufgezeigt werden, um dann jeweils Handlungsempfehlungen in drei konkreten Feldern einer demografieorientierten Personalpolitik zu geben.

\section{Herausforderung eines strategi- schen Personalmanagements}

In Zuge des voranschreitenden demografischen Wandels in der öffentlichen Verwaltung, der mit einem starken Anstieg des Durchschnittsalters einhergeht, gibt es vor allem Herausforderungen für die Leistungsfähigkeit der individuell alternden Mitarbeitenden in drei Hauptbereichen: (a) der körperlichen Leistungsfä-

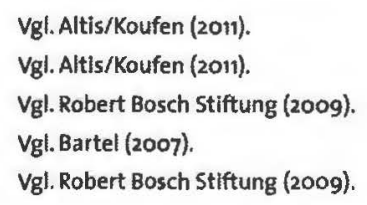




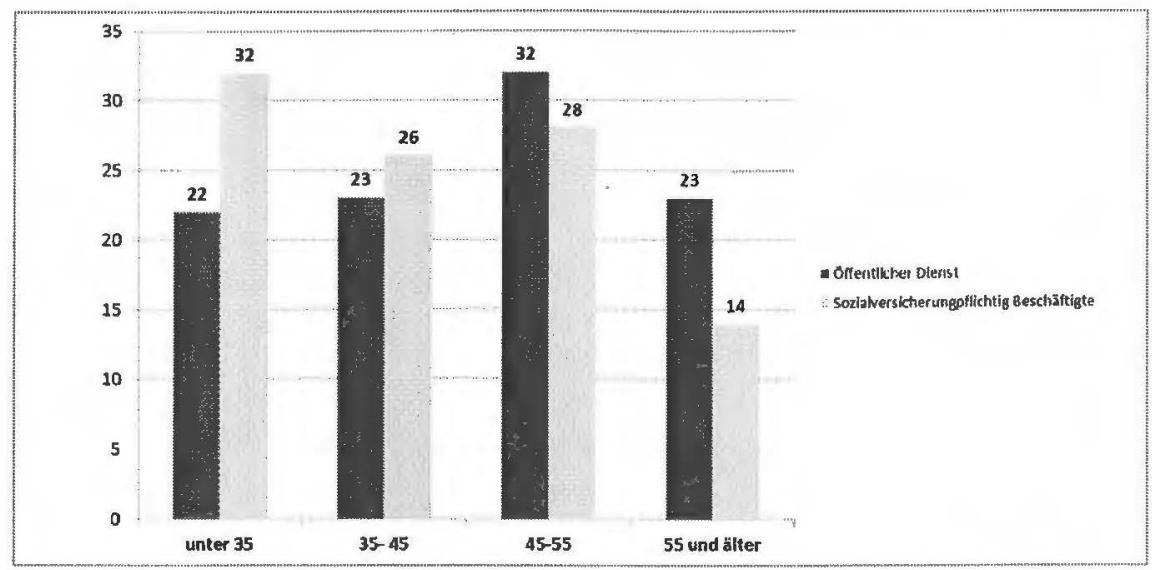

Abb. 1: Vergleich des prozentualen Anteils von Altersgruppen zwischen öffentlichem Dienst und sonstigen sozialversicherungspflichtig Beschäftigten. Quelle: Altis/Koufen (2011)

higkeit, (b) der Qualifikation und (c) der Motivation. ${ }^{6}$ In den nächsten Abschnitten sollen diese drei Bereiche jeweils kurz beleuchtet werden und dann mit einem (a) präventiven Gesundheitsmanagement, (b) einer auf lebenslanges Lernen ausgerichteten Personalentwicklung und (c) eines lebensphasenorientierten Karrieremanagements, jeweils konkrete Handlungsfelder zur Bewältigung der Herausforderungen aufgezeigt werden. Abbildung 2 gibt einen Überblick wie die Herausforderungen und Handlungsfelder schematisch in Verbindung stehen.

\section{Körperliche Leistungsfähigkeit und Alter(n)}

Häufig ist in den Köpfen vieler Angestellter und leider auch bei Führungskräften noch ein starkes Defizitmodells des Alter(n)s verankert. Steigendes Alter wird fast zwangsweise mit geringerer Belastbarkeit, höherer gesundheitlicher Anfälligkeit und insgesamt geringer Produktivität in Verbindung gebracht und diese Altersvorurteile haben häufig einen starken Einfluss auf die Entwicklungschancen von älteren Mitarbeitenden.

Unbestritten hat der Alterungsprozess natürlich Einfluss auf die körperliche Leistungsfähigkeit. Die beiden Hauptmerkmale der körperlichen Leistungsfähigkeit, die maximale Sauerstoffaufnahme und die Belastbarkeit des Muskel- und Skelettsystems hängen zumindest teilweise mit dem Alter der Mitarbeitenden zusammen. So erreicht die Sauerstoffaufnahme im Durchschnitt mit 30 Jahren ihren $\mathrm{Hö}$ hepunkt und die maximale Beweglichkeit des Muskel- und Skelettsystems geht späzurück. ${ }^{7}$ Allerdings gibt es in beiden Bereichen starke interindividuelle Schwankungen zwischen Mitarbeitenden des gleichen Alters. So können durch gezieltes Training und Präventionsınaßnahmen die körperlichen Leistungswerte eines 65-jährigen Mitarbeitenden durchaus besser sein als die eines 45-jährigen Mitarbeitenden. ${ }^{8}$ Auch sportwissenschaftliche Untersuchungen von großen Marathonveranstaltungen haben gezeigt, dass ca. 25 Prozent der über 65-70-jährigen Teilnehmenden in der Lage sind, besser abzuschneiden Deshalb nuss der Prozess des Alterns bis in das sechste Lebensjahrzehnt keinen starken Einfluss auf die körperliche Leistungsfähigkeit der Mitarbeitenden haben. ${ }^{10}$ Hinzu kommt, dass insbesondere in der Dienstleistungserstellung der öffentlichen Verwaltung häıfig keine Tätigkeiten vorliegen, in denen eine maximale körperliche Leistungsfähigkeir notwendig ist. Vielmehr kann insbesondere bei Bürotätigkeiten auch mit einer leicht zurückgehenden körperlichen Konstitution noch eine durchatis gure Leistungserstellung erreicht werden.

Nichtsdestotrotz nimmt aber die absolut Zahl der krankheitsbedingten Fehltage durch den Anstieg des Dirrchschnittsalters der Belegschaften auch in den öffentlichen Verwaltungen zu. ${ }^{11}$ Das ist vorwiegend darauf zurückzuführen, dass ältere Mitarbeitenden zwar nicht häufiger, aber länger ausfallen. Solche langen Ausfallzeiten zu vermeiden, ist demnach eine der zentralen Bestandteile eines präventiven Gesundheitsmanagements, wie es im nächsten Abschnitt vorgestellt wird. Hierbei ist be- testens ab einem Alter von 45-50 Jahren als Teilnehmende zwischen 20-54 Jahre.? sonders darauf zu achten, dass neben physischen auch auf psychische Fehlbelastungen geachtet wird, da psychische Faktoren den stärksten Anstieg als Ursache von Fehltagen in den letzten Jahren verzeichnen. ${ }^{12}$ Ebenso haben psychologische Faktoren nicht nur eine direkte Wirkung auf psychologischen Beschwerden (zum Beispiel Burnout), sondern auch viele körperliche Beschwerden, wie Rückenleiden oder Herz-Kreislauferkrankungen sind häufig psychisch bedingt. ${ }^{13}$

\section{Einsatz eines präventiven Gesundheitsmanagements}

Um den beschriebenen Herausforderungen in Zusammenhang zwischen Alter und körperlicher Konstitution erfolgreich $\mathrm{zu}$ begegnen, muss in den öfentlichen Verwaltungen stärker als heute ein präventives Gesundheitsmanagement als eine zentrale Komponente eines strategischen Personalmanagements im demografischen Wandel eingerichtet werden. Das Gesundheitsmanagement sollte vor allem nicht vollständig auf einem "Präsentismus" ausgerichtet werden. Dies bedeutet, dass sich die Maßnahmen des Gesundheitsmanagements nicht nur auf diejenigen Mitarbeitenden beschränken sollten, die schon einen starken Anstieg der Fehlzeiten aufzeigen. Eine Verminderung von Belastungen von schon physisch eingeschränkten Mitarbeitenden und das Wiedereingliederungsmanagement von Mitarbeitenden mit langen Fehlzeiten sind natürlich wichtige Maßnahmen bei einer alternden Belegschaft, greifen alleine aber zu kurz, da sie nur die Symptome und nicht die Ursachen steigender Fehlzeiten bekämpfen. Deshalb ist es wichtig, dass ein präventives Gesundheitsmanagement nicht nur auf ältere, schon leistungseingeschränkte Mitarbeitende ausgerichtet wird, sondern auf Mitarbeitende aller Altersgruppen in den Dienststellen und Betrieben. Vermeidung von physischen (z.B. ergonomische

\footnotetext{
6 Vgl. Bruch/Kunze/Boehm (2011). Vgl. Ilmarinen (2001).

Vgl. Ilmarinen (2001).

Vgl. Leyk et al. (2009)

10 Vgl. Petrenz (1999)

1 Vgl. Bundesministerium des Innern (2012).

12 Vgl.AOK (2012).

13 Vgl.Slegrist/Peters, 2011
} 


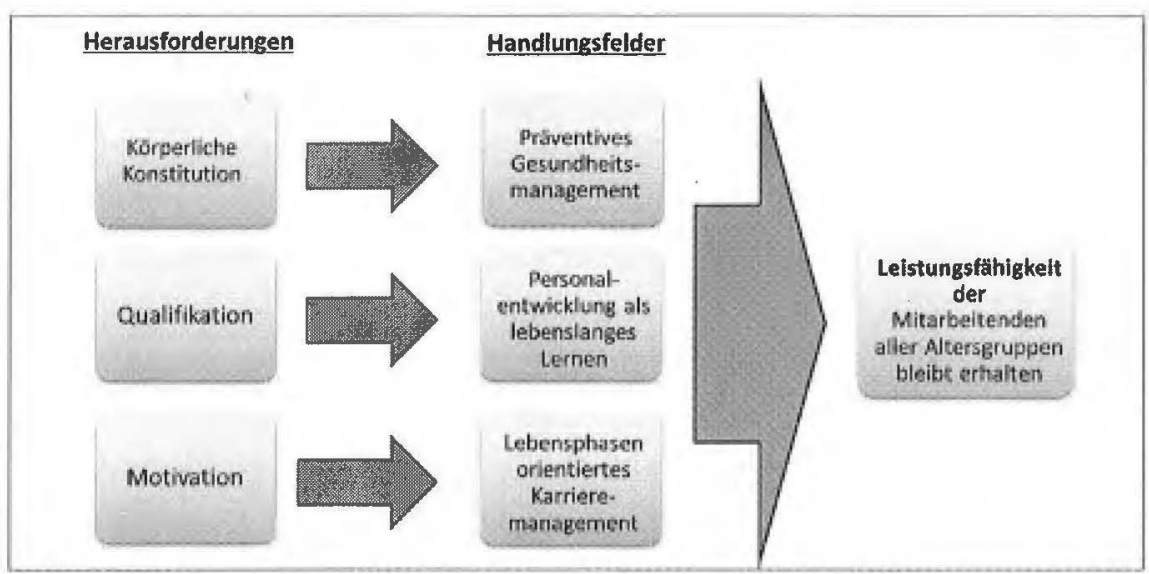

Abb. 2: Herausforderungen und Handlungsfelder für ein strategisches Personalmanagement in der öffentlichen Verwaltung

Anpassung der Arbeitsplätze) und psychischen Fehlbelastungen (Vermeidung von Stress und emotionalen Konflikten) im betrieblichen Alltag sollten deshalb für die gesamte Belegschaft angestrebt werden. Dadurch kann es dann gelingen, nicht nur zukünftige Fehlzeiten zu vermeiden, sondern auch die Leistungsfähigkeit der anwesenden Mitarbeitenden zu erhöhen.

Empirische Studien zeigen, dass neben einer individuellen Gesundheitsförderung auch das Führungsverhalten eine entscheidend Rolle für den Erhalt der Arbeitsfähigkeit spielt. So konnte in einer Längsschnittstudie gezeigt werden, dass die Arbeitsfähigkeit zwischen dem 40, und 60. Lebensjahr sogar ausgebaut werden konnte, wenn gleichzeitig eine individuelle Gesundheitsförderung, ergononische Maßnahmen und eine alter(n)sgemäßes Führungsverhalten vorhanden waren. ${ }^{14}$ Gesundheitsmanagement sollte deshalb auch in der öffentlichen Verwaltung als eine zentrale Führungsaufgabe und nicht nur als der Aufgabenbereich des Personalmanagements betrachtet werden. Gesundheitsfaktoren könnten so z.B. als ein Bestandteil für Zielvereinbarungen aller Altersgruppen verwendet werden. In Tätigkeitsbereichen mit besonders hohem Potenzial für Fehlzeiten (z.B. Pflegedienste) können zusätzlich auch Instrumente wie der Arbeitsfähigkeitsindex zum Einsatz kommen. ${ }^{15}$ Dieser Index erhebt als ein zukunftsgerichtetes Instrumentarium die heutige und zukünftige Arbeitsfähigkeit und ermöglicht es so, eventuell auch mit dem Hinzuziehen eines Betriebsarztes, präventive Maßnahmen zun Erhalt der selbigen einzuleiten.
Schließlich sollte ein präventives Gesundheitsmanagement auch immer Teil der strategischen Gesamtausrichtung der Organisations- und Verwaltungseinheit sein. Dazu gehört zum Beispiel, dass die nachhaltige Gesundheit der Mitarbeitenden als ein zentrales strategisches Ziel definiert wird und auch die Top-Führungskräfte dies so kommunizieren und ihr persönliches Handeln entsprechend anpassen. Nur so kann es gelingen, eine Gesundheitskultur in der ganzen Organisation zu etablieren, die nachhaltig zu einem Erhalt der körperlichen Leistungsfähigkeit aller Altersgruppen beiträgt.

\section{Qualifikation und Alter(n)}

Auch für die kognitive Leistungsfähigkeit und Qualifikation von älteren Mitarbeitenden gibt es ein ähnlich vorherrschendes Defizitmodelle wie für die körperliche Leistungsfähigkeit. Demnach sind ältere Mitarbeitende nur schwer in der Lage, sich neues Wissen anzueignen und haben starke Widerstände gegen organisationale Veränderungsprozesse. In der vorhandenen empirischen Forschung haben sich aber viele dieser postulierten Defizitmodelle als nicht haltbar herausgestellt. ${ }^{16}$

Vielmehr ist auch für die Entwicklung der Qualifikation im Alter eine differenziertere Betrachtung notwendig. Hier lohnt sich insbesondere die Unterscheidung zwischen zwei Dimensionen von kognitiven Fähigkeiten, der fluiden und kristallinen Intelligenz ${ }^{17}$. Die fluide Intelligenz beinhaltet die Kompetenzen, die vor allem für die Verarbeitung von neuem Wissen und Informationen notwendig ist. Durch sie ist es möglich, schnell neue Sachverhalte zu erfassen und Aufgaben ohne den Rückgriff auf bestehendes Wissen zu lösen. Nach dem derzeitigen Stand der psychologischen Forschung erreicht diese Art der intellektuellen Kompetenz schon mit ca. 27 Jahren ihren Höhepunkt. ${ }^{18} \mathrm{Al}$ lerdings zeigen andere Studien, dass auch fluide Fähigkeiten bis ins achte Lebensjahrzehnt durch spezifisches Training zumindest stabil gehalten werden können. ${ }^{19}$

Kristalline Intelligenz hingegen beschreibt praktisches, erfahrungsbasiertes Wissen und intellektuelle Fähigkeiten. Sie beinhaltet häufig routinierte Problemlösungskompetenzen und ist meistens während des Arbeitslebens einem stetigen Anstieg unterworfen. Insgesamt ist sie stark sozialisationsabhängig und wird damit zum Beispiel stark durch das Arbeitsumfeld, in dem man sich bewegt, beeinflusst. Im Allgemeinen ist es möglich, dass ältere Mitarbeitende ihre zurückgehende fluide Problemlösungsfähigkeit durch einen Anstieg der kristallinen erfahrungsbasierten Fähigkeiten ausgleichen können. ${ }^{20}$ Hinzu kommt, dass für viele Tätigkeiten insbesondere im Bereich der öffentlichen Leistungserstellung, vor allem kristallines und erfahrungsbasiertes Wissen benötigt wird. Im Speziellen sind dies T'ätigkeiten, die entweder Routinetätigkeiten mit einem hohen $\mathrm{Maß}$ an Wiederholungen und einfachen geistigen Anforderungen (z.B. einfache Sachbearbeitertätigkeiten in einfachen und mittleren Dienst) erfordern, oder auch Aufgaben, die eine stark wissensbasierte Urteilsfindung ohne groBen Zeitdruck erfordern (z.B. richterliche Tätigkeiten oder Professoren). Zum Teil können in diesen Bereichen ältere Mitarbeitende bessere Ergebnisse als jüngere erzielen. ${ }^{21}$ Lediglich bei Arbeitsaufgaben, die mit einer permanenten Wissensverarbeitung und starken körperlichen Anstrengungen verbunden sind (z.B. Call-Center-Tätigkeiten, Fließbandarbeit) weisen

\footnotetext{
14 Vgl. llimarinen/Tempel (2002).

$15 \mathrm{Vgl}$.lllmarinen/Tempel (2002).

16 Vgl. Posthuma/Campion (2009).

$17 \mathrm{Vgl.Catell}(1987)$.

18 Vgl. Kanfer/Ackermann (2000).

19 Vgl. Ball et al. (2002).

$20 \mathrm{Vgl}$. Bruch et al. (2010).

21 Vgl. Warr (1996).
} 
ältere Mitarbeitende häufig Defizite auf. Diese sind aber in Bereich der Tätigkeiten im offentlichen Dienst eher selten anzutreffen.

Wichtig zum Erhalt der Qualifikation und Kompetenz im Alter ist vor allem, dass für alle Altersgruppen im öffentlichen Bereich kontinuierlich neve Qualifikationen aufgebaut werden. Aufgrund der zurückgehenden fluiden Intelligenz sind ältere Mitarbeitende zwar gewissen Beschränkungen bein Erwerb von neuen Wissen in Laufe ihres Erwerbslebens unterworfen. Dies sollte aber nicht zu einem generellen Ausschluss von Weiterbildungsmaßnahmen führen, wie es in der Ver- ihre Lemfähigkeit und Qualifikationsbreite sehr stark reduziert wird. ${ }^{22} \mathrm{Z}_{\text {war }}$ ist die Weiterbildungsquote insbesondere von Beamten im Vergleich zu anderen Erwerbstätigen relativ hoch, aber auch hier nehmen 30 Prozent der Mitarbeitenden an keinen Weiterbildungen teil. Auch ist insgesamt die Weiterbildungsquote unter älteren Mitarbeitenden am niedrigsten, auch wenn in den letzten Jahren dort aufgrund der fortlaufenden öffentlichen Diskussion ein Aufwärtstrend zu erkennen ist. ${ }^{23}$ Wichtig ist auch, dass jetzt aufgrund der Thematik des demografischen Wandels nicht der Fehler begangen wird, Weiterbildungsmaßnahmen von Älteren auf Kosten der jüngeren Beschäftigten aus-

\section{"Trainings- und Weiterbildungsmaßnahmen sollten nicht zu stark auf eine Altersgruppe fokussiert werden, sondern eine generelle Kultur des lebenslangen Lernens in den Dienststellen und Verwaltungen sollte aufgebaut werden."}

waltungspraxis häufig zu finden ist. Wie ein solches altersunabhängiges Weiterbildungskonzept im Rahmen des lebenslangen Lernens in die öffentlichen Verwaltungen integriert werden kann, wird im folgenden Abschnitt dargestellt.

\section{Personalentwicklung als lebenslanges Lernen}

Wie im vorherigen Abschnitt festgehalten wurde, ist es auch bei älteren Mitarbeitenden sinnvoll noch in Weiterbildungsund Trainingsmaßnahinen zu investieren. Generell gilt es den "Teufelskreis" zu durchbrechen, der besagt das Ältere nicht mehr motiviert sind $\mathrm{zu}$ lemen und sie damit systematisch von Weiterbildungsmaßnahmen ab einem bestimnten Alter ausgeschlossen werden, was wiederum dazu führt, dass ihre Qualifikation sinkt. Inshesondere wird die Lernkompetenz älterer Mitarbeitenden dadurch eingeschränkt, dass sie über Jahre und Jahrzehnte auf bestimmte Arbeitsvorgänge und Verfahren beschränkt sind und damit den). Anderseits zeigt die empirische Forschung, dass ältere Mitarbeitende andere Erwartungshaltungen und Herangehensweisen an Trainingsmaßnahmen als jüngere Mitarbeitende haben. ${ }^{24}$ Für sie spielt es keine ausschlaggebende Rolle, durch die Weiterbildung instrumentelle, langfristige Ziele wie eine Gehaltserhöhung oder ein Karrierefortschritt zu erreichen. Vielmehr streben sie danach, möglichst praxisnah weitergebildet $z u .^{25}$ Wenn Weiterbildungsveranstaltungen in dieser Form auf die Präferenzen und die kristallinen Stärken der älteren Beschäftigten angepasst werden (mehr 'lraining on-the-jol, meln. selbstorganisiertes Lernen), dürften sie deutlich effektiver und wirksamer auch in der öffentlichen Verwaltungen werden.

\section{Motivation und Alter(n)}

Bei der Betrachtung der Motivation von älteren Beschäftigten in der öffentlichen Verwaltung ist es zunächst einmal interessant festzustellen, dass ältere Mitarbeitende, die noch im Arbeitsleben verblieben sind, im Durchschnitt die höchsten Werte für die Arbeitszufriedenheit und die Identifikation mit ihrem Arbeitgeber von allen untersuchten Altersgruppen haben. ${ }^{26}$ Es ist also ein Irrglaube, älteren Mitarbeitenden generell eine niedrigere Motivation in Erwerbsleben zuzusprechen. Allerdings macht die neuste organisationspsychologische Forschung deutlich, dass es veränderte Motive zur Arbeitsmotivation von Mitarbeitenden verschiedener Altersgruppen gibt. ${ }^{27}$ Wïhrend jüngere Mitarbeitende eine starke Wachstums- und Karriereorientierung bei ihren beruflichen Zielen haben, geht es bei älteren Mitarbeitenden vor allem darum, entstehende Defizite auszugleichen. ${ }^{28}$ Wie schon in den vorherigen Abschnitten zur körperlichen und kognitiven Fähigkeiten beschrieben, können ältere Mitarbeitende mit den richtigen Strategien oft bis zur Verrentungsgrenze potenzielle Defizite ausgleichen oder sogar überwinden. Trotzden führt diese

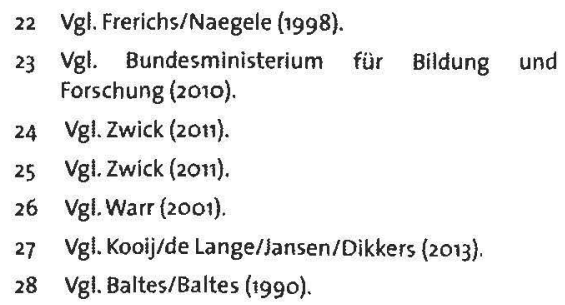


unterschiedliche motivationale Prioritätensetzung dazu, dass Mitarbeitende verschiedener Altersgruppen unterschiedliche Ziele als wichtig im Erwerbsleben erachten. Während jüngere Mitarbeitende nach langfristigen instrumentellen Zielen, wie Aufstiegsmöglichkeiten und besserer Bezahlung streben, sind ältere Mitarbeitende eher an kurzfristigen emotionalen Zielen, wie persönlicher Anerkennung und guten sozialen Beziehungen am Arbeitsplatz, interessiert.

Hinzu kommt, dass neben diesen unterschiedlichen Motiven auch die unterschiedlichen Lebensphasen der Mitarbeitenden berücksichtigt werden sollten. Junge Mütter und Familienväter brauchen z.B. andere Arbeits- und Karrieremodelle als Mitarbeitende mittleren Alters (ca. diese derzeit in Anspruch ${ }^{29}$ ), ist in diesem Zusammenhang nicht besonders zielführend. Im Speziellen ist dies der Fall, da sie fast ausschließlich in geblockter Form Anwendung findet, dass heißt Mehrarbeit für eine bestiminte Zeit und dann ein früherer Ubergang in den Ruhestand. Zum einen manifestiert dieses Instrument stark das Defizitmodell des Alters in der Organisation, da implizit für die älteren $\mathrm{Be}$ schäftigten der frühzeitige Eintritt in den Ruhestand als Hauptziel ausgegeben wird und sie damit als nicht mehr notwendige Ressource für den Arbeitsprozesse angesehen werden. Durch die ausschließliche geblockte Anwendung dieser Maßnahmen werden zudem auch ihre potenziellen Vorteile ausgehebelt. Durch eine schrittweise Reduzierung der Arbeitszeit wäre zum Beispiel die Vermeidung von Überlastun-

\section{"Hauptziel eines an Lebensphasen orientierten Karrieremanagements muss es sein, Mitarbeitenden aller Altersgruppen konkrete Entwicklungsziele in ihrem Berufsleben aufzuzeigen."}

\begin{abstract}
45 Jahre), deren Kinder gerade zu Hause ausgezogen sind und die deshalb wieder größere Kapazitäten hätten, um sich wieder stärker beruflich einzubringen. Diese Punkte sollten in einem lebensphasenorientierten Führungs- und Karrieremanagement in der öffentlichen Verwaltung berücksichtigt werden, wie es im folgenden Abschnitt vorgestellt wird.
\end{abstract}

\section{Lebensphasenorientiertes Karrieremanagement}

Ein lebensphasenorientiertes Karrieremanagement soll den veränderten Motiven und Bedürfnissen, insbesondere älterer Beschäftigten in der öffentlichen Verwaltung Rechnung tragen. Die Altersteilzeit, die derzeit fast als alleiniges personalpolitisches Instrument für ältere Mitarbeitende in öffentlichen Dienst eingesetzt wird (mehr als 28 Prozent der über 55-jährigen in der öffentlichen Verwaltung nehmen gen bei erfahrenen Mitarbeitenden und damit ein Weiterarbeiten bis zum Renteneintrittsalter möglich. Weiterhin könnte durch ein schrittweises Ausscheiden aus dem Beruf auch das Einarbeiten von jüngeren Kollegen und ein erfolgreicher impliziter Wissenstransfer ermöglicht werden.

Hauptziel eines an Lebensphasen orientierten Karrieremanagements muss es hingegen sein, Mitarbeitenden aller Altersgruppen noch konkrete Entwicklungsziele in ihrem Berufsleben aufzuzeigen. Damit kann es gelingen, den Zeitpunkt in dem aus der Wachstumsorientierung in beruflichen Leben eher eine Orientierung auf die Kompensation von Defiziten wird, so weit wie möglich hinauszuzögern. Aktuelle Studien, an denen wir gerade forschen, zeigen, dass durch die Sinnhaftigkeit, die Mitarbeitende in ihrer Tätigkeit sehen, sich das subjektive Alter stark von dem chronologischen Alter unterscheiden kann. Mitarbeitende, die sich jünger fühlen, haben demnach eine weitaus höhere Leistungsfähigkeit, da sie sich subjektiv noch in der Wachstums- und Karrierephase befinden. Aber auch für Mitarbeitende, die schon ihren beruflichen Zenit in Behörden und Verwallungseinheiten erreicht haben, sollten noch lebensphasengerechte Angebote zur weiteren Entwicklung gegeben werden. Dies muss nicht immer ein weiterer hierarchischer Aufstieg sein. Vielmehr können auch eine weitere fachliche Entwicklung oder eine stärkere Rolle als Coach oder Mentor potenzielle Entwicklungsziele sein. Solche eher sozialorientierten Aufgaben kommen auch den Bedürfnissen von älteren Mitarbeitenden nach einer emotionalen und sozialen Wertschätzung entgegen. Mögliche weitere Entwicklungsschritte können zum Beispiel in Karrieremanagementseminaren für Mitarbeitenden als einem bestimnten Alter (zum Beispiel 45+) angeboten werden. In diesen wird es den erfahrenen Mitarbeitenden ermöglicht, aktiv über ihre derzeitige Situation und weitere Entwicklungsmöglichkeiten $\mathrm{zu}$ reflektieren und andere Karriereziele als einen vorzeitigen Ruhestand anzustreben.

Neben einem aktiven Karrieremanagement sollten die Karriere- und Entwicklungsperspektiven auch auf die jeweilige Lebensphase der Arbeitnehmer angepasst werden. Wichtig ist, dass eine Flexibilisierung nicht nur in Alter in Form von flexiblen Übergängen in den Ruhestand, sondern während der gesamten Erwerbsbiographie, möglich ist. Bewährt hat sich zum Beispiel das Modell des Lebensarbeitszeitkontos, mit denen Mehrarbeit über längere Zeiträume angespart werden kann. Diese Überstunden können dann je nach Lebenssituation in Sabaticals, Bildungsurlaub oder in einen früheren Rentenbeginn eingetauscht werden. Das Land Hessen hat ein solches Modell zum Beispiel für alle seine Beamten im Jahre 2012 eingeführt. ${ }^{30}$ Ebenso vorbildlich im Bereich des öffentlichen Diensts ist die lebensphasenorientierte Personalpolitik der Bundesagentur für Arbeit. In vier Phasen werden hier flexible personalpolitische

\footnotetext{
$29 \mathrm{Vgl.Altis/Koufen} \mathrm{(2011).}$

30 Vgl. Verwaltung Hessen (2013).
} 
Maßnnahmen für alle Altersgruppen angeboten, die sowohl Familiengründung und Karriere für jüngeren Mitarbeitende als auch einen „zweiten Karrierestart" für Mitarbeitende mittleren Alters erlauben. ${ }^{31}$ Diese beiden Beispiele zeigen, dass ein aktives lebensphasenorientiertes Personalmanagement auch heute schon erfolgreich in öffentlichen Organisationen praktiziert wird.

\section{Fazit - ein strategisches Personalmanagement im demo- grafischen Wandel lohnt sich}

Der vorliegende Beitrag hat skizziert, wie ein strategisches Personalmanagement in drei Kernbereichen - Gesundheitsmanagement, Personalentwicklung, lebensphasenorientiertes Karrierenanagement - zur Bewältigung des demografischen Wandels in öffentlichen Verwaltungen ausgestaltet werden kann. Empirische Studien, die wir mit einer Vielzahl von privaten und öffentichen Organisationen durchgefuihrt haben, machen deutlich, dass ein alterssensibles Personalmanagement nicht nur gesamtgesellschaftlich wünschenswert ist, sondern auch klare Leistungsimplikationen hat. So konnten wir zeigen, dass in 93 klein- und mittelständischen Unternehmen die Gesanıtorganisationsleistung zunimmt, wenn Personalmanagenıentmaßnahmen auf unterschiedliche Altersgruppen abgestimmt werden. ${ }^{32}$ Auch in einer zweiten Studie konnten wir zeigen, dass ein aktives Demografiemanagement positiv mit der Arbeitszufriedenheit und dem Commitment der Mitarbeitenden zusammenhängt und gleichzeitig die emotionale Erschöpfung aller Altersgruppen reduziert. ${ }^{33}$ Es besteht wenig Zweifel, dass diese Ergebnisse auf den öffentlichen Sektor zu übertragen sind und dort die Effizienz und Effektivität des Verwaltungshandelns, trotz eines steigenden Durchschnittsalters der Belegschaften, durch ein besseres Personalmanagement gesteigert werden könmen.

\footnotetext{
$31 \mathrm{Vgl}$. Inqua (2013).

$32 \mathrm{Vgl}$. Boehm/Kunze/Bruch (2013)

33 Vgl. Kunze/Bruch (2012).
}

\section{Literatur}

Altis, A./Koufen S. (2011): Entwicklung der Beschäftigung im öffentlichen Dienst, in: Statistischen Bundesamt, Wirtschaft und Statistik, 11/2011: S. 1111-1116.

AOK (2012): Fehlzeitenreport 2012, Berlin.

Bartel, W. (2007): Demographischer Wandel und Personalflexibilität von Kommunen in Ostdeutschland, Polen und Westdeutschland, in: Sackmann, R./Bartel, W./Kopycka, K./Rademacher, C. (Hrsg.): Strategien zur Bewältigung des demographischen Wandels im öffentlichen Sektor. Interkommunale Vergleiche in Ost* und Westdeutschland sowie in Polen, SFB 580 Mitteilung, Heft 2, Jena, S. 50-95.

Ball, K./Berch, D.B./Helmers, K.F./Jobe, J.B./ Leveck, M.D./Marsicke, M./Morris, J.N./Rebock, G.W./Smith, D.M./Tennenstedt, S. L./Unverzagt, F.W./Willis, S.L. (2002): Effects of cognitive training interventions with older adults: a randomized controlled trial, in: Journal of the American Medical Association, Vol. 18, S. 2271-228?.

Baltes, P. B./Baltes, M. M. (1990): Psychological perspectives on successful aging: The model of selective optimization with compensation, in Baltes, P. B./Baltes, M. M. (Eds.), Successful aging: Perspectives from the behavioral sciences, S. 1-34, Cambridge.

Boehm, S./Kunze, F./Bruch, H. (2013). A spotlight on age-diversity climate - how age related human resource management practices might lead to organizational performance. Personnel Psychology, im Druck.

Bruch, H./Kunze, F./Boehm (2010): Generationen erfolgreich Führen. Konzepte und Praxiserfahrungen zum Management des demographischen Wandels. Wiesbaden, Gabler.

Bundesministerium des Innern (2012): Gesundheitsförderungsbericht 2011 der unmittelbaren Bundesverwaltung - einschließlich Fehlzeitstatistik, Berlin.

Bundesministerium für Bildung und Forschung (2012): Weiterbildungsverhalten in Deutschland, Berlin.

Cattell, R. B. (1987). Intelligence: Its structure, growth, and action, Amsterdam.

Frerichs, F./Naegele G. (1998): Strukturwandel des Alters und Arbeitsmarktentwicklung - Perspektiven der Altererwerbsarbeit im demogra phischen und wirtschaftlich-kulturellen wandel, in: Clemens W./Backes. G. M. (Hrsg.): Altern und Gesellschaft, Opladen, S. 237-256.

Kunze, F./Bruch, H. (2012): Aktives Demografiemanagement, in: Zeitschrift für Führung und Organisation, Vol. 81, S. 397-402.

Ilmarinen, J. (2001): Aging workers, in: Occupational and Environmental Medicine Vol. 58,5 546-552.

Ilmarinen, J./Tempel, H. (2002): Arbeitsfähigkeit 2010. Was können wir tun, damit wir gesund bleiben?, Berlin.

Inqua (2013): Leistungs- und Innovationsfähigkeit sichern - Das praxisorientierte Personalmanagement der Bundesagentur für Arbeit, www.inqa.de/DE/Lernen-Gute-Praxis/Top-100Gute-Unternehmenspraxis/Personalfuehrung/ Bundesagentur-fuer-Arbeit-Leistungs-undInnovationsfaehigkeit-sichern.html (besucht 8.07.2013)
Kanfer, R./Ackermann, P. L. 2004. Aging, adult development, and work motivation. Academy of Management Review, Vol. 29, S. 440-457.

Kooij, D. T./de Lange, A. H./Jansen, P. G./ Dikkers, J. S. (2013). Beyond chronological age. Examining perceived future time and subjective health as age-related mediators in relation to work-related motivations and well-being, in: Work \& Stress, Vol. 27, S. 88-105.

Leyk, D./Erley, O./Gorges, W./Ridder, D./Rüther, T./Wunderlich, M./Sievert, A./Essfeld, D./ Piekarski, C./Erren, T. (2009). Performance, training and lifestyle parameters of marathon runners aged $20-80$ years: results of the PACEstudy, in: International Journal of Sports Medicine, Vol. 30, 5. 360-365.

Petrenz J. (1999): Alter und berufliches Leistungsvermögen, in: Gussone, M./Huber, A./ Morschäuser, M./Pertrenz, J. (Hrsg): Ältere Arbeitnehmer, S.63-99, Frankfurt am Main.

Posthuma, R. A./Campion, M. A.(2009): Age Stereotypes in the Workplace: Common Stereotypes, Moderators, and Future Research Directions, in: Journal of Management, Vol. 35, S. 158188.

Robert Bosch Stiftung (2009): Demographieorientierte Personalpolitik in der öffentlichen Verwaltung, Stuttgart.

Siegrist, 1./Peter, R. (1996): Assessing chronically stressfull experience at work: Implications for prevention, in: BAUA (Hrsg.): Tagungsbericht 11, Occupational Health and Safety Aspects of Stress in Modern Workplaces, Berlin.

Verwaltung Hessen (2013): Einführung eines Lebensarbeitszeltskonto, http://verwaltung.hes sen.de/irj/HMdl_Internet?cid=74dagagd1b1037 ce7dbo205fee97do82 (besucht 8.07.2013)

Warr, P.B. (1996): Younger and Older Workers, in: Warr, P.B (Hrsg.): Psychology at Work, Harmondsworth, S. 308-332.

Warr, P. (2001): Age and work behaviour: Physical Attributes, cognitive abilities, knowledge, personality traits and motives, in: International Review of Industrial and Organizational Psychology, Vol. 16, S, 1-34.

Zwick, T. (2011): Why training older employees is less effective, in: ZEW-Research Discussion Paper, 11-046. 